\title{
Is there a correlation between venlafaxine therapy during pregnancy and a higher incidence of necrotizing enterocolitis?
}

\author{
Markus Treichel, Katharina Schwendener Scholl, Ulf Kessler, Alexander Joeris, \\ Mathias Nelle
}

Berne, Switzerland

Background: Novel antidepressant drugs are increasingly used by women of child bearing age. However, potentially harmful effects on fetus and newborn remain unknown.

Methods: Case report and literature review.

Results: We present preterm twins whose mother was treated with venlafaxine, a nonselective serotonin reuptake inhibitor, throughout pregnancy until delivery. The twins developed neonatal necrotizing enterocolitis.

Conclusion: The question whether there might be a correlation between maternal serotonin reuptake inhibitor therapy and neonatal necrotizing enterocolitis is discussed.

World J Pediatr 2009;5(1):65-67

Key words: antidepressive agents; necrotizing enterocolitis; nonselective serotonin reuptake inhibitor; pregnancy; venlafaxine

\section{Introduction}

$\sqrt{ }$ enlafaxine $\left(\right.$ Efexor $\left.^{\mathbb{R}}\right)$ is a nonselective serotonin reuptake inhibitor (nSSRI). It is supposedly a potent serotonin and norepinephrine reuptake inhibitor and a weak inhibitor of dopamine reuptake. It is commonly used in the therapy of depression and panic disorders. Venlafaxine is metabolized in the liver and is mainly eliminated by the kidneys. In adults the average elimination half life of venlafaxine is 5 hours and that of

Author Affiliations: Department of Neonatology (Treichel M, Schwendener Scholl K, Nelle M) and Department of Surgical Pediatrics (Kessler U, Joeris A), Children's Hospital, University of Berne, Berne, Switzerland

Corresponding Author: Ulf Kessler, Department of Surgical Pediatrics, Inselspital, 3010 Berne, Switzerland (Tel: +41 31 6329223; Fax: +41 31 6329292; Email: ulf.kessler@insel.ch)

doi:10.1007/s12519-009-0013-6

(C)2009, World J Pediatr. All rights reserved. its active metabolite O-desmethylvenlafaxine 11 hours. In neonates the elimination half life is unknown.

Reproduction studies in rats and rabbits given venlafaxine at doses up to 2.5 to 4 times the maximum recommended human daily dose did not reveal teratogenicity. ${ }^{[1]}$ When rats were given the maximum dose during pregnancy through weaning, there was a decrease in pup weight and an elevated number of stillbirths and pup deaths during the first 5 days of lactation. ${ }^{[1]}$

Einarson et $\mathrm{al}^{[2]}$ compared 150 pregnant women using venlafaxine to 150 women exposed to selective serotonin reuptake inhibitors (SSRIs) and 150 women exposed to nonteratogenic agents. There were no significant differences in the rate of abortions, gestational age at birth, live births, birth weights, and major malformations. Withdrawal symptoms of newborn infants after exposure to SSRI and nSSRI are well-described. ${ }^{[3,4]}$

\section{Case report}

We present dichorial diamniotic twins who both developed necrotizing enterocolitis (NEC) on the 6th day of life. They were delivered by cesarean section at a gestational age of $33+2$ weeks due to persistent vaginal bleeding and contractions secondary to placenta praevia marginalis. The first two trimesters of pregnancy were uneventful. The mother was hospitalized for the first time in the 31st week of pregnancy because of vaginal bleeding. Because of an infection with Chlamydia trachomatis, she was treated with azithromycin (zithromax ${ }^{\circledR}$ ) for 4 days. She received betamethasone (celestone chondrose ${ }^{\circledR}$ ) 12 mg twice in 24 hours to enhance fetal lung maturity. Furthermore the mother was treated with venlafaxine (Efexor $^{\circledR}$ ) throughout pregnancy at a single dosage of $75 \mathrm{mg}$ per day until delivery because of depression.

Twin A was born with a birthweight of $1700 \mathrm{~g}$ (15th percentile). He had Apgar scores of 6 at 1 minute, 7 at 5 minutes, and 8 at 10 minutes. The umbilical arterial cord $\mathrm{pH}$ was 7.22.

The baby was intubated in the first hour of life 
because of respiratory insufficiency and supplemental oxygen requirement. He was extubated successfully on the second day of life. Feeding was started on the first postnatal day with maltodextrin ${ }^{\circledR}$ and breast milk. Enteral feeds with breast milk and preterm formula were gradually increased by about $20 \mathrm{ml} / \mathrm{kg}$ per day until the feeding volume reached $100 \mathrm{ml} / \mathrm{kg}$ per day on the 5 th postnatal day.

On the 6th day of life, the child showed signs of necrotizing enterocolitis (NEC) with abdominal distension and bloody stool. Conservative management consisted of withholding oral feeds for 3 days and intravenous antibiotics, amikacine and amoxicilline for 5 and 7 days, respectively. Apart from clostridium difficile toxin in the stool specimen, blood and stool cultures were negative. The abdominal X-ray revealed no signs of pneumoperitoneum or pneumatosis intestinalis (Bell's stage I). Feeds were well-tolerated afterwards.

Twin B was born with a birthweight of $1980 \mathrm{~g}$ (30th percentile). He had Apgar scores of 4 at 1 minute, 5 at 5 minutes, and 8 at 10 minutes. The umbilical arterial cord $\mathrm{pH}$ was 7.24 .

Twin B was also ventilated on his first day of life for respiratory insufficiency and supplemental oxygen requirement. Enteral feeds were also started on the first day of life with maltodextrin ${ }^{\circledR}$ and breast milk. As in twin A, enteral feeds with breast milk and preterm formula were gradually increased by about $20 \mathrm{ml} / \mathrm{kg}$ per day until the feeding volume reached $80 \mathrm{ml} / \mathrm{kg}$ per day on the 5 th postnatal day.

On the 6th day, he developed abdominal distension, signs of peritonitis, bloody stool and gastric residuals. Conservative therapy with intravenous antibiotics (amikacine and amoxicilline) and stop of oral feeding was initiated. The abdominal X-ray showed intestinal pneumatosis, but no pneumoperitoneum (Bell's stage IIb). Because of further clinical deterioration under conservative management he underwent surgery on the 10th day of life. A long segment bowel necrosis with several perforations was found. Terminal ileum, right colon and the proximal transverse colon were resected, and enterostomy was performed.

Culture of peritoneal fluid, blood and stool remained negative. Feeding was started on the third postoperative day. On the 22nd day of life the baby had to undergo a second operation because of stomal stenosis. The stoma was closed at the age of five months when he weighed $3000 \mathrm{~g}$. At laparotomy we found colonic stenosis. At several levels a complete luminal obliteration of the remaining transverse colon and of the proximal part of the descending colon was seen and confirmed by histological examination. The remaining transverse and proximal descending colons were resected and an anastomosis between ileum and descendent colon was created. The postoperative course was uneventful and the patient was discharged 10 days after stoma closure.

\section{Discussion}

We presented dichorial diamniotic twins with a gestational age of $33+2$ weeks who both developed necrotizing enterocolitis on the 6th day of life. Their mother was treated with venlafaxine, a nonselective serotonin reuptake inhibitor, throughout pregnancy until delivery. The question is whether therapy with venlafaxine during pregnancy is associated with a higher incidence of NEC or not. To date no clear association has been described in the literature, although neonatal withdrawal symptoms are well described. ${ }^{[3,4]}$ Both twins required mechanical ventilation, although they had mild signs of hyaline membrane disease only. Surfactant therapy was not necessary and ventilatory requirements were moderate. It might be speculated that respiratory insufficiency could be secondary from maternal venlafaxine therapy. However only two cases of respiratory disease have been reported in association with venlafaxine treatment in adults..$^{[5,6]}$

We found a case report describing two term neonates whose mothers were treated with a selective serotonin reuptake inhibitor (paroxetine) throughout pregnancy ${ }^{[7]}$ The babies developed NEC on the 3rd and 4 th day of life respectively. The uncommon occurrence of NEC in term neonates raises the question of whether serotonin reuptake inhibitors, selective or not, increase the risk for microcirculatory failure, possibly causing NEC.

SSRI reduces platelet serotonin by inhibiting its uptake. This occasionally causes platelet dysfunction and bleeding. ${ }^{[8]}$ Stiskal et $\mathrm{al}^{[7]}$ speculated that increased platelet function as a rebound phenomenon may predispose the neonate to a hypercoagulable state. Since platelet activating factor plays a central role in the pathogenesis of NEC, disturbed platelet function is supposed to be mainly involved in disease progression. ${ }^{[9]}$

Cocaine functions as a reuptake inhibitor of norepinephrine and dopamine amongst other neurotransmitters. Maternal cocaine use causes vasoconstriction that can result in placental abruption and perinatal stroke. ${ }^{[10]}$ This assumption is mainly based on case reports, but the study of Heier et $\mathrm{al}^{[10]}$ reported that $17 \%$ of full-term infants born to cocaine users had evidence of cortical infarction. Furthermore, cocaine exposure can lead to diminished gut bloodsupply and thereby contribute to the pathogenesis of 
NEC. Two studies confirmed the contributory role of cocaine abuse during pregnancy in the etiology, time of onset, and extent of neonatal NEC. ${ }^{[11,12]}$

Nonselective serotonin reuptake inhibitors as venlafaxine may have a similar mechanism of action to cocaine. It might be speculated that the inhibition of norepinephrine reuptake causes vasoconstriction in the intestine and hence ischemia that can lead to necrosis.

In the present case, prematurity certainly is an independent and essential risk factor for NEC. The correlation between enterocolitis and the venlafaxine therapy is speculative but possible and should be kept in mind when treating pregnant women and particularly when caring for newborns exposed to venlafaxine in utero.

\section{Funding: None.}

Ethical approval: Not needed.

Competing interest: None declared.

Contributors: Treichel M wrote the main body of the article under the supervision of Schwendener Scholl $\mathrm{K}$ and Kessler U. Kessler U and Joeris A provided advice on medical aspects. Nelle $\mathrm{M}$ is the guarantor.

\section{References}

1 Product information. Effexor ${ }^{\circledR}$ (venlafaxine hydrochloride) Tablets. Wyeth Pharmaceuticals Inc., 2008. www.wyeth.com/ content/showlabeling.asp?id=99 (accessed July, 2008).

2 Einarson A, Fatoye B, Sarkar M, Lavigne SV, Brochu J, Chambers $\mathrm{C}$, et al. Pregnancy outcome following gestational exposure to venlafaxine: a multicenter prospective controlled study. Am J Psychiatry 2001;158:1728-1730.

3 Costei AM, Kozer E, Ho T, Ito S, Koren G. Perinatal outcomes following third trimester exposure to paroxetine. Arch Pediatr Adolesc Med 2002;156:1129-1132.

4 De Moor RA, Mourad L, ter Haar J, Egberts AC. Withdrawal symptoms in a neonate following exposure to venlafaxine during pregnancy. Ned Tijdschr Geneeskd 2003;147:1370-1372.

5 Melien O, Skaali T, Myhr K, Brors O. Venlafaxine and asthma. Nord J Psychiatry 2005;59:538-540.

6 Turner RC, Nelson JE, Roberts BT, Gillam DM. Venlafaxine associated interstitial penumonitis. Pharmacotherapy $2005 ; 25$ 626-629.

7 Stiskal JA, Kulin N, Koren G, Ho T, Ito S. Neonatal paroxetine withdrawal syndrome. Arch Dis Child Fetal Neonatal Ed 2001;84:F134-135.

8 Nair GV, Gurbel PA, O'Connor CM, Gattis WA, Murugesan SR, Serebruany VL. Depression, coronary events, platelet inhibition and serotonin reuptake inhibitors. Am J Cardiol 1999;84:321-323.

9 Muguruma K, Gray PW, Tjoelker LW, Johnston JM. The central role of PAF in necrotizing enterocolitis development. Adv Exp Med Biol 1997;407:379-382.

10 Heier LA, Carpanzano CR, Mast J, Brill PW, Winchester P, Deck MD. Maternal cocaine abuse: the spectrum of radiologic abnormalities in the neonatal CNS. AJNR Am J Neuroradiol 1991;12:951-956.

11 Lopez SL, Taeusch HW, Findlay RD, Walther FJ. Time of onset of necrotizing enterocolitis in newborn infants with known prenatal cocaine exposure. Clin Pediatr (Phila) 1995;34:424-428.

12 Czyrko C, Del Pin CA, O'Neill JA Jr, Peckham GJ, Ross AJ 3rd. Maternal cocaine abuse and necrotizing enterocolitis: outcome and survival. J Pediatr Surg 1991;26:414-418.

Received September 24, 2007 Accepted after revision May 7, 2008 